\title{
Elevation of arachidonoylethanolamide levels by activation of the endocannabinoid system protects against colitis and ameliorates remote organ lesions in mice
}

\author{
XIAOLIN ZHAO ${ }^{1,2 *}$, PENG LIANG ${ }^{1,3^{*}}$, JIN LIU $^{1,3}$, HAIXIA JIANG $^{1,4}$, \\ XIAOSHUAI FAN ${ }^{1}$, GUO CHEN ${ }^{1,3}$ and CHENG ZHOU ${ }^{1,3}$ \\ ${ }^{1}$ Laboratory of Anesthesia and Critical Care Medicine, Translational Neuroscience Center, West China Hospital, \\ Sichuan University; ${ }^{2}$ West China School of Pharmacy, Sichuan University; ${ }^{3}$ Department of Anesthesiology, \\ West China Hospital, Sichuan University, Chengdu, Sichuan 610041; ${ }^{4}$ Department of Anesthesiology, \\ The First Affiliated Hospital of Kunming Medical University, Yunnan, Kunming 650221, P.R. China
}

Received November 30, 2016; Accepted June 29, 2017

DOI: $10.3892 / \mathrm{etm} .2017 .5222$

\begin{abstract}
The endocannabinoid system (ECS) is a potential pharmaceutical target for the treatment of inflammatory bowel diseases (IBDs). The aim of this study was to explore the effects of activation of the ECS on IBD and the associated neural inflammation-induced disruption of the blood-brain barrier (BBB). In a mouse model of trinitrobenzene sulfonic acid-induced colitis, the inhibition of fatty acid amide hydrolase with URB597 elevated the arachidonoylethanolamide concentration of the colon. Macroscopic alterations of the colons were evaluated, and the 7-day survival rate of mice was analyzed. BBB integrity was assessed using a dye tracer method, and the cognitive function of mice was examined using a fear-conditioning test. URB597 treatment significantly reduced macroscopic alterations of the colon, decreased the mortality rate, and protected the integrity of the $\mathrm{BBB}$ in the mice $(\mathrm{P}<0.05)$. No significant changes were observed in the cognitive functions of the mice $(\mathrm{P}>0.05)$; therefore, the neuroprotective effect of ECS in this colitis model requires further investigation. Activation of the ECS was efficient in ameliorating colitis and increasing the survival rate of the
\end{abstract}

Correspondence to: Dr Guo Chen, Department of Anesthesiology, West China Hospital, Sichuan University, 37 Guo Xue Xiang, Chengdu, Sichuan 610041, P.R. China

E-mail: grace_chenguo@hotmail.com

Dr Cheng Zhou, Laboratory of Anesthesia and Critical Care Medicine, Translational Neuroscience Center, West China Hospital, Sichuan University, 88 South Keyuan Road, Chengdu, Sichuan 610041, P.R. China

E-mail: zhouc@163.com

${ }^{*}$ Contributed equally

Key words: URB597, fatty acid amide hydrolase, endocannabinoid system, colitis, blood-brain-barrier integrity mice, and reducing remote organ changes induced by colitis. The results suggest that modulation of the ECS is a potential therapeutic approach for IBDs and the associated remote organ lesions.

\section{Introduction}

As lipid mediators, endocannabinoids are known to be involved in a variety of physiological and pathological process, including protection of the cardiovascular system, depression and various inflammatory responses (1-3). Due to their significance in physiological functions, endocannabinoids have attracted considerable attention since being identified. Endogenously generated from cell membrane lipid precursors when required, these lipid mediators exert their functions through binding to and activating two $G$ protein-coupled receptors, namely cannabinoid (CB) receptors 1 and 2, which are distributed throughout the peripheral and central nervous system (4). The actions of endocannabinoids are terminated via cellular uptake by membrane transporters and hydrolysis by specific enzymes, such as fatty acid amide hydrolase (FAAH) and monoacylglycerol lipase (5). Endocannabinoids and CB receptors, together with their biosynthesis and degradation enzymes, constitute the endocannabinoid system (ECS).

There is evidence to suggest that the ECS is an efficient regulating system in inflammatory conditions, and closely correlated with the pathogenesis and advancement of inflammation bowel diseases (IBDs) (6-9). In a clinical setting, the activation of $\mathrm{CB}$-relevant receptors has been reported to help with controlling colitis symptoms and relieving anxiety in patients with IBDs (10). CB receptor gene knockout has been demonstrated to increase the susceptibility of mice to trinitrobenzene sulfonic acid (TNBS)-induced colitis (11). The inhibition of endocannabinoid hydrolytic enzymes was found to be efficient in attenuating gut inflammation in a murine colitis model $(12,13)$. Thus, it appears that the ECS may exert beneficial effects on IBD therapy.

In addition to exerting protective effects against inflammatory colitis, the ECS has also been indicated to be beneficial 
in ameliorating systemic inflammation and nervous system lesions (14). Activation of the ECS decreased pro-inflammatory cytokine levels in mouse models of inflammation and sepsis $(15,16)$, and also ameliorated neural inflammation and attenuated behavioral alterations in mice with Huntington's disease (17). Furthermore, elevation of endogenous arachidonoylethanolamide (AEA, also known as anandamide) levels via the inhibition of FAAH protected rats against aging-associated inflammatory responses in the hippocampus (18), attenuated Toll-like receptor 3-induced neural inflammatory responses (19), and ameliorated posttraumatic brain injury in mice (20). This evidence strongly suggests a neural protective role of the ECS.

In the present study, the aim was to explore the role of the ECS on colitis-concomitant organ lesions in the central nervous system. Since it has been demonstrated that TNBS-induced acute colitis results in endoxemia and neural inflammation, due to a breakdown of the intestinal barrier and increased permeability of the colon (6-9), the contribution of ECS to organ-protection in TNBS-induced colitis was investigated in the study. Blood-brain-barrier (BBB) integrity was also evaluated. To explore any alteration of cognitive functions, fear conditioning tests (FCTs) were performed to assess the learning and memory ability of the mice.

\section{Materials and methods}

Animals. Male adult C57BL/6 mice ( $\mathrm{n}=215,8-12$ weeks; 20-25 g) were obtained from the Laboratory Animal Center of Sichuan University (Sichuan, China), and housed in a room (humidity, $40-60 \%)$ at constant temperature $\left(22 \pm 2^{\circ} \mathrm{C}\right)$ with a 12-h light/dark cycle, and free access to water and food. The Animal Use and Care Committee of Sichuan University, following the guidelines of the Care and Use of Laboratory Animals of China Association for Laboratory Animal Science, approved the experimental protocols conducted using animals.

Induction of colitis. TNBS (Sigma-Aldrich; Merck KGaA, Darmstadt, Germany) was applied to induce colitis, as previously described $(12,21,22)$. In brief, mice were fasted for $16 \mathrm{~h}$, and anesthetized with $1 \%$ isoflurane. TNBS $(100 \mathrm{mg} / \mathrm{kg}$ in saline-ethanol, 50:50, v/v) was infused into the colon using a cannula placed $4 \mathrm{~cm}$ from the anus. Mice in the control group received the same dosage of vehicle (saline-ethanol, 50:50, $\mathrm{v} / \mathrm{v})$. Following the infusion, the tail of each mouse was held in a vertical position for $\geq 1 \mathrm{~min}$, for the homogenous distribution and retention of the TNBS enema.

Drug administration and group assignment. URB597 (Sigma-Aldrich; Merck KGaA) was dissolved in a mixed solvent (ethanol-Tween-80 saline, 1:1:18, v/v/v) as previously described (12). Mice were randomized into four groups: Control group $(n=40)$, colitis group $(n=67)$, URB597 pre-treated group $(n=54)$ and URB597 post-treated group $(n=54)$. In the pre-treated group, the mice received URB597 injections [2 mg/kg subcutaneously (s.c.), twice daily (b.i.d.)] at 4 and $2 \mathrm{~h}$ prior to the induction of colitis; while in the post-treated group, the mice received URB597 $(2 \mathrm{mg} / \mathrm{kg}$, s.c., b.i.d.) following the induction of colitis and every day thereafter until mortality. Mice in the control group and the

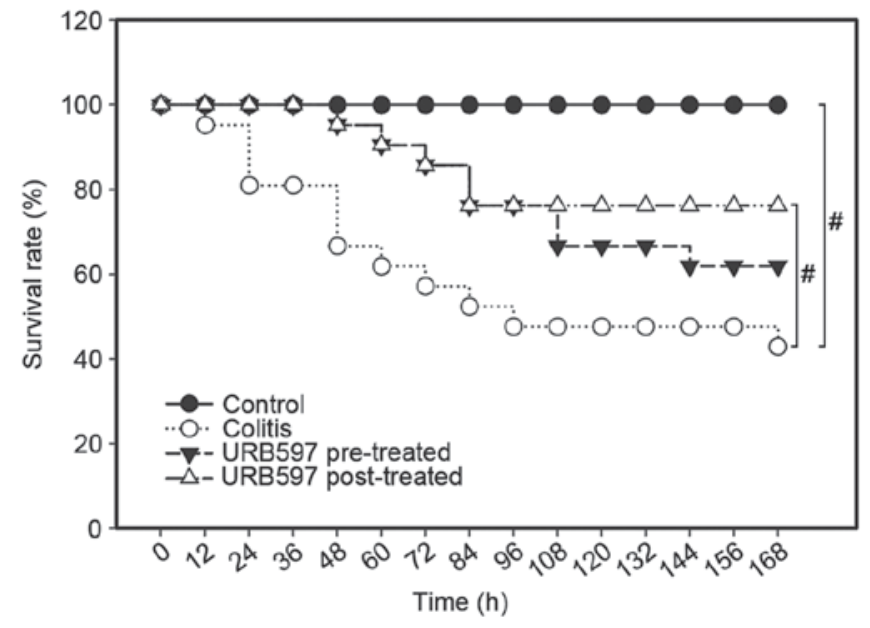

Figure 1. Survival rate of mice in the four groups was recorded every $12 \mathrm{~h}$. The pre-treated group received URB597 subcutaneously prior to the induction of colitis using trinitrobenzene sulfonic acid, while the post-treated group was treated with URB597 twice daily following colitis induction. The sample sizes were, control group $(n=10)$; colitis group $(n=21)$; URB597 pre-treated group $(n=21)$; and URB597 post-treated group $(n=21)$. Data were analyzed using the log-rank sum test followed by a pairwise over strata test. The data indicated that URB597 post-administration significantly decreased colitis-induced mortality in the mice $\left({ }^{\#} \mathrm{P}<0.05\right)$. Although URB597 pre-administration also decreased the mortality rate, the reduction was not statistically significant.

colitis model group received the mixed solvent instead of URB597. Subcutaneous administration was applied in order to maintain a stable endocannabinoid concentration according to the previous study (12). Euthanasia was conducted for 8-10 mice on days 1, 3 and 7 after the induction of colitis, and colons were removed for subsequent analysis. The tissues used for macroscopic evaluation of colitis injury were put into formalin to be fixed. Other tissue used for measurement of BBB permeability and endocannabinoids were preserved in liquid nitrogen until use.

The present study comprises three experimental sections and all four groups were evaluated in each section. In the first section, the mortality rate was determined for each group in order to investigate the effects of URB597 on the survival of the mice. In the second section, measurements of colitis pathology and BBB permeability on days 1, 3 and 7 were made. In the third section, the cognitive function of the mice was assessed and endocannabinoids concentrations were measured. In each section of the study, mice were randomly assigned into the groups.

Macroscopic evaluation of colitis injury. Mice were sacrificed on days 1, 3 and 7 after the induction of colitis. Mice were sacrificed via an intraperitoneal injection of an overdose of pentobarbital sodium. Colons were removed quickly and longitudinally incised and carefully washed with ice-cold normal saline. Then the colons were put into formalin to be fixed. Lengths of colons were measured by macroscopic evaluation. Briefly, the formalin-fixed tissue was dehydrated with grades of alcohol and cleared in xylene. The tissues were then embedded in paraffin prior to examination using light microscopy. Macroscopic alterations of the colons were evaluated by a researcher blinded to the treatment the mice 


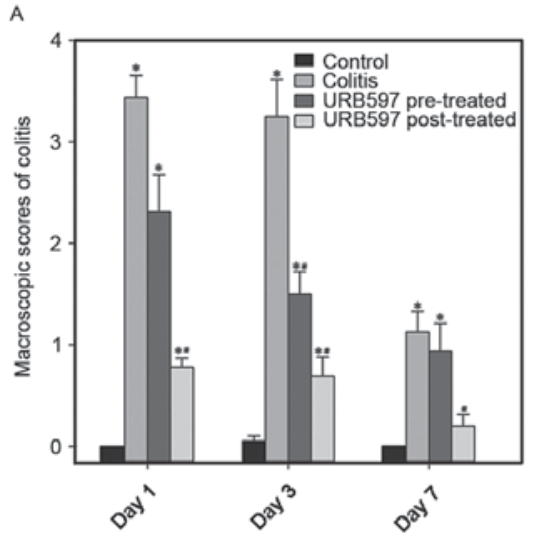

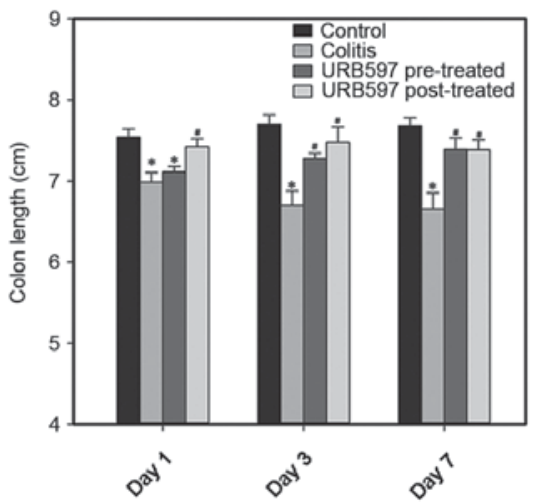

C

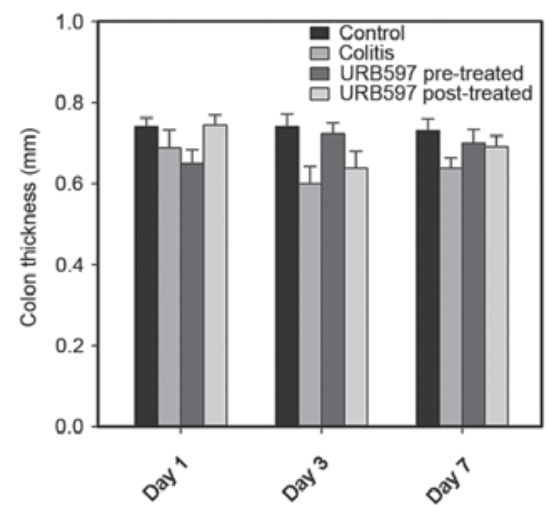

Figure 2. Activation of the ECS via the inhibition of FAAH ameliorated TNBS-induced colitis. URB597 was administered to mice prior to or following the induction of colitis using TNBS. The control and colitis groups received an intraperitoneal injection of mixed solvent vehicle. (A) Macroscopic scores, (B) lengths and (C) thicknesses of the colons. The number of animals analyzed in each group was 8-10. Values are expressed as the mean + standard error of the mean, and were analyzed using the Kruskal-Wallis test followed by Dunn's multiple comparison post hoc tests. * $<0.05$ vs. time-matched control group; ${ }^{\#} \mathrm{P}<0.05$ vs. time-matched colitis group. ECS, endocannabinoid system; FAAH, fatty acid amide hydrolase; TNBS, trinitrobenzene sulfonic acid.

had received, using an established semi-quantitative scoring system (21): Ulcer $(0.5$ points for each $0.5 \mathrm{~cm})$; colonic shortening ( 1 point, $\geq 15 \%$; 2 points, $\geq 25 \%$, based on the average length of colons from the control group); every additional presence of hemorrhage, diarrhea or fecal blood added 1 point to the score. Colon length was measured using a vernier caliper. Intestinal wall thickness was measured by light microscopy examination.

$B B B$ permeability measurement. A dye tracer extravasation method was applied for the detection of BBB integrity (20). Evans blue (2\%, $2 \mathrm{ml} / \mathrm{kg}$, intravenously) was administered 30 min prior to euthanasia. Following euthanasia, the brains were removed quickly and homogenized in dimethylformamide (DMF). The homogenates were incubated at $37^{\circ} \mathrm{C}$ overnight for dye extraction. The tissue suspension was centrifuged at $1,800 \mathrm{x} \mathrm{g}$ for $10 \mathrm{~min}$ at room temperature, and the optical density of the supernatant was measured at $655 \mathrm{~nm}$ with a spectrophotometer. A linear standard curve of Evans blue in DMF was applied in order to calculate the concentration in the samples. Data are expressed in units of Evans blue $(\mu \mathrm{g}) /$ brain tissues $(\mathrm{g})$.

Evaluation of cognitive behavior using FCTs. On the basis of the results of $\mathrm{BBB}$ integrity, it was speculated that the cognitive functions of the mice may be affected by neuro-inflammation. Evaluation of cognitive behavior was performed using FCTs, following previously described guidelines (23). In the FCTs, cue tone tests and context tests were conducted to detect sound-related and context-related aversive memory respectively. Tests were performed at 8,9 and 10 days after model induction.

Measurement of endocannabinoids using liquid chromatography-mass spectrometry (LC-MS). Following the FCT, the concentrations of endocannabinoids were determined using LC-MS. The endocannabinoids determined were as follows: AEA, 2-arachidonoylglycerol (2-AG), palmitoylethanolamide (PEA) and arachidonic acid (AA). Briefly, the mice were anesthetized under $3 \%$ isoflurane and sacrificed by decapitation. Colons were removed quickly and snap frozen and stored at $-80^{\circ} \mathrm{C}$ until use. Samples were weighed and transferred to tubes under ice-cold conditions. The tubes contained $1 \mathrm{ml} 0.1 \%$ formic acid and acetonitrile with deuterated endocannabinoids, and $3 \mathrm{ml}$ ethylacetate/hexane (9:1). The mixture was homogenized and then centrifuged at $4,600 \times \mathrm{g}$ for $15 \mathrm{~min}$ at $4^{\circ} \mathrm{C}$. The upper organic phase was evaporated to dryness in a stream of nitrogen and the residue was reconstituted in $100 \mu \mathrm{l}$ acetonitrile. Following centrifugation for $15 \mathrm{~min}$ at $14,000 \mathrm{x} \mathrm{g}\left(4^{\circ} \mathrm{C}\right)$, a $5-\mu 1$ aliquot of sample was injected into the LC-MS/MS system for analysis (Agilent 6400; Agilent Technologies, Inc., Santa Clara, CA, USA). The chromatographic conditions used were as previously described (24).

Statistical analysis. Data are expressed as the mean \pm standard error of the mean and were analyzed using SPSS 22.0 software (IBM Corp., Armonk, NY, USA). The macroscopic scores of colons were analyzed using rank-sum testing with a Kruskal-Wallis test, followed by Dunn's multiple comparison post hoc tests. Survival analysis was performed using a log-rank sum test followed by pairwise over strata tests. Other experimental data were analyzed using one-way analysis of variance with Bonferroni post hoc tests when the data obeyed normal distribution characteristics; otherwise, data were analyzed using a Kruskal-Wallis test with Dunn's multiple comparison post hoc tests. $\mathrm{P}<0.05$ was considered to indicate a statistically significant difference.

\section{Results}

Administration of URB597 improves the survival rate of mice with TNBS-induced colitis. TNBS-induced colitis caused a severe systemic inflammatory response and led to the mortality of mice. The 7-day survival rate of the mice was recorded to explore the potential effects of the ECS on mortality rate. As the results indicate (Fig. 1), URB597 treatment significantly increased the survival rate of the mice, compared with that in the colitis model group $(\mathrm{P}<0.05)$. During the first $72 \mathrm{~h}$, the survival rate in the URB597 
Table I. Macroscopic scores of the colon (mean \pm standard error of the mean).

\begin{tabular}{llll}
\hline & \multicolumn{3}{c}{ Macroscopic score of the colon } \\
\cline { 2 - 4 } Group & Day 1 & Day 3 & Day 7 \\
\hline Control & $0.00 \pm 0.00$ & $0.05 \pm 0.05$ & $0.00 \pm 0.00$ \\
Colitis & $3.44 \pm 0.22^{\mathrm{a}}$ & $3.25 \pm 0.37^{\mathrm{a}}$ & $1.13 \pm 0.21^{\mathrm{a}}$ \\
URB597 pre-treated & $2.31 \pm 0.37^{\mathrm{a}}$ & $1.50 \pm 0.22^{\mathrm{a}, \mathrm{b}}$ & $0.94 \pm 0.27^{\mathrm{a}}$ \\
URB597 post-treated & $0.78 \pm 0.09^{\mathrm{a}, \mathrm{b}}$ & $0.69 \pm 0.19^{\mathrm{a}, \mathrm{b}}$ & $0.20 \pm 0.11^{\mathrm{b}}$
\end{tabular}

${ }^{\mathrm{a}} \mathrm{P}<0.05$ vs. control group; ${ }^{\mathrm{b}} \mathrm{P}<0.05$ vs. colitis group.

Table II. Concentration of Evans blue in brain tissue (mean \pm standard error of the mean).

\begin{tabular}{lccc}
\hline & \multicolumn{3}{c}{ Evans blue content $(\mu \mathrm{g} / \mathrm{g})$} \\
\cline { 2 - 4 } Group & Day 1 & Day 3 & Day 7 \\
\hline Control & $386 \pm 0.37$ & $3.90 \pm 0.30$ & $3.86 \pm 0.45$ \\
Colitis & $7.06 \pm 0.65^{\text {a }}$ & $5.18 \pm 0.37$ & $5.45 \pm 0.52$ \\
URB597 pre-treated & $5.62 \pm 0.56^{\mathrm{b}}$ & $5.34 \pm 0.62$ & $5.18 \pm 0.53$ \\
URB597 post-treated & $4.31 \pm 0.43^{\mathrm{b}}$ & $4.44 \pm 0.29$ & $5.17 \pm 0.31$ \\
\hline
\end{tabular}

${ }^{\mathrm{a}} \mathrm{P}<0.05$ vs. control group; ${ }^{\mathrm{b}} \mathrm{P}<0.05$ vs. colitis group.

pre-treatment (86\%) and post-treatment (86\%) groups was markedly higher than that in the colitis group (57\%). The survival rate of mice in the TNBS model group was only $43 \%$ on day 7, while that in the URB597 post-treated group was $76 \%(\mathrm{P}<0.05)$ and that in the URB597 pre-treated group was $62 \%(P>0.05)$, indicating that ECS was effective in increasing the survival rate of mice with colitis.

URB597 attenuates TNBS-induced colitis. The intrarectal TNBS enema induced a reproducible acute colitis murine model, characterized by an increase in the macroscopic scores of the colons (Fig. 2A and Table I). The macroscopic score in the TNBS model group was significantly higher than that in the control group (day $1,3.44 \pm 0.22$ vs. $0.00 \pm 0.00, \mathrm{P}<0.05$; day $3,3.25 \pm 0.37$ vs. $0.05 \pm 0.05$; $\mathrm{P}<0.05$ ). Reductions in colon length were also critical markers for colitis (Fig. 2B). Colon thickness was similar among groups (Fig. 2C). These data indicate that the TNBS-ethanol enema successfully induced acute colitis.

To study the impact of the ECS on TNBS-induced colitis, URB597, a selective FAAH inhibitor, was administered to treat colitis. The macroscopic scores of the colons were decreased in the URB597 treatment groups (Fig. 2A and Table I), particularly for the URB597 post-treated group, which was significantly different from the colitis model group (day 1, $0.78 \pm 0.09$ vs. $3.44 \pm 0.22$; day $3,0.69 \pm 0.19$ vs. $3.25 \pm 0.37$; $\mathrm{P}<0.05)$. The shortening of the colon length was also attenuated significantly in the URB597 treatment groups compared with the colitis group (Fig. 2B). Colon thickness was similar

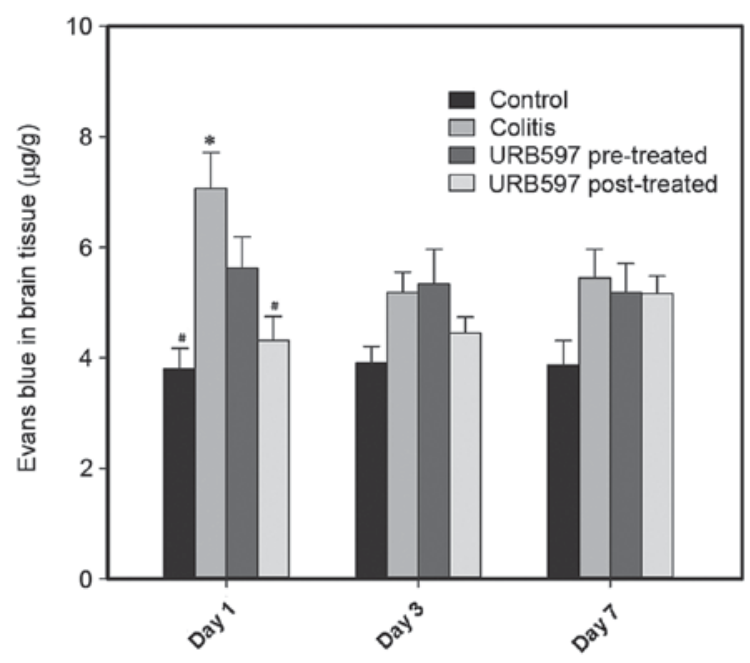

Figure 3. BBB integrity was assessed on days 1, 3 and 7. The number of animals analyzed in each group was 8-10. Data are presented as mean + standard error of the mean, and were analyzed using Kruskal-Wallis test with Dunn's multiple comparison post hoc test. " $\mathrm{P}<0.05$ vs. time-matched control group; ${ }^{\#} \mathrm{P}<0.05$ vs. time-matched colitis model group. $\mathrm{BBB}$, blood-brain barrier.

among groups (Fig. 2C). These data indicate a beneficial role of ECS in this model.

Stimulation of the ECS exerts a protective effect on $B B B$ integrity. Colitis induces disruption of the intestinal barrier, and further leads to systemic inflammation and a neural inflammatory response. The effect of neural inflammation on the integrity of the BBB was detected by assessing the penetration of Evans blue dye into the brain tissue. As shown in Fig. 3 and Table II, increased Evans blue entry into the brain tissue was observed in the colitis group $(7.06 \pm 0.65 \mu \mathrm{g} / \mathrm{g})$ compared with the control group $(3.86 \pm 0.37 \mu \mathrm{g} / \mathrm{g})$ on day 1 $(\mathrm{P}<0.05)$, indicating the destruction of BBB integrity during the occurrence of colitis. URB597 pre- and post-treatment exerted protective effects by maintaining BBB integrity (pre-treated group, $5.62 \pm 0.56 \mu \mathrm{g} / \mathrm{g}$; post-treated group, $4.31 \pm 0.43 \mu \mathrm{g} / \mathrm{g}$ ) on day 1 , and post-treatment exerted a significantly stronger protective effect on the BBB compared with pre-treatment $(\mathrm{P}<0.05)$. These results indicated that ECS stimulation improved BBB integrity under the inflammatory conditions of colitis. On days 3 and 7, the Evans blue content in the brain tissues was reduced, suggesting that the BBB integrity recovered to a normal level in the mice of the colitis group.

Colitis-associated lesions do not impair the cognitive functions of mice. To further explore whether cognitive functions were affected by neural inflammation, the cognitive behavior of the mice was evaluated using FCTs. Reduced freezing time percentages and increased freezing latency are considered to be indicative of memory decline, which suggests cognitive function damage. Freezing episodes may indirectly reflect rodent activity. As shown in Fig. 4, there was no significant difference in these indices among the groups $(\mathrm{P}>0.05)$. Although a trend toward cognitive dysfunction was detected in the colitis group, no statistical significance was detected 


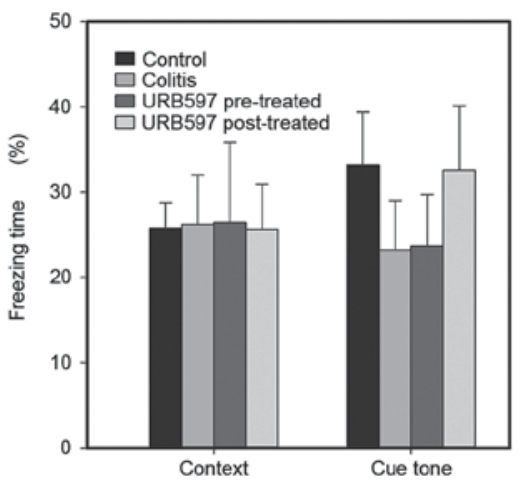

B

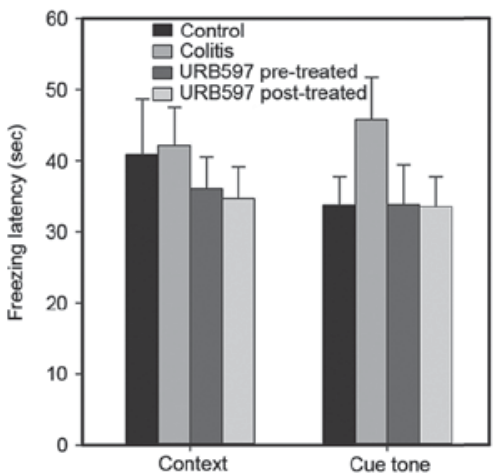

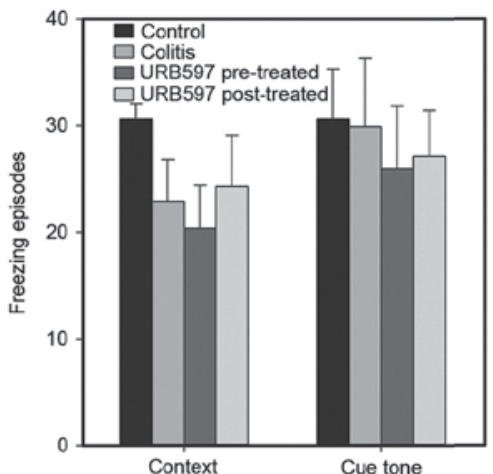

Figure 4. Fear conditioning test was conducted on days 8, 9 and 10 to evaluate the memory decline of the mice. Percentage of (A) freezing time, (B) freezing latency, and (C) number of freezing episodes, as three important indices, were analyzed using the Kruskal-Wallis test with Dunn's multiple comparison post hoc test. Data are expressed as the mean + standard error of the mean. The number of animals analyzed per group was 10 .
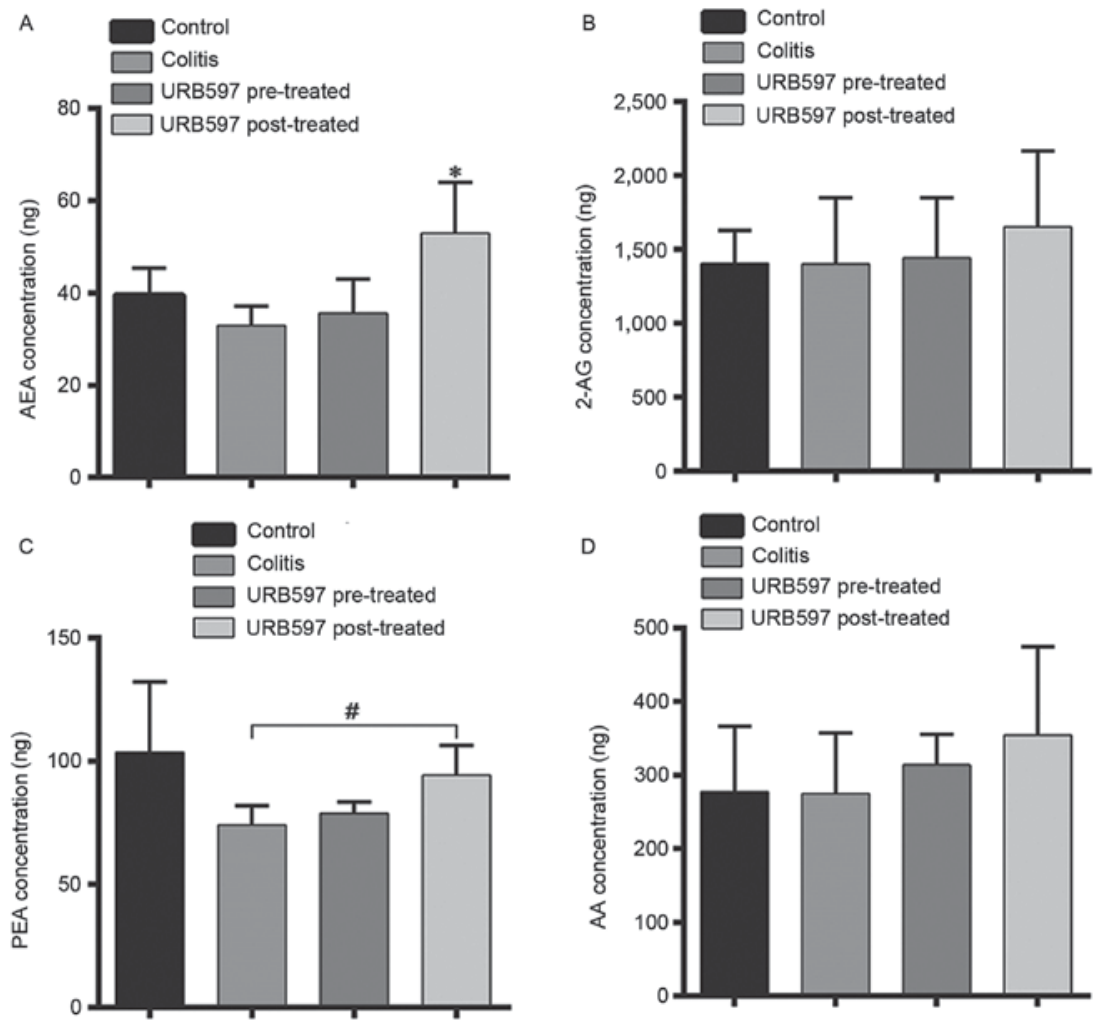

Figure 5. Endocannabinoid concentrations. The concentrations of (A) AEA, (B) 2-AG, (C) PEA and (D) AA were determined by gas chromatography-mass spectrometry. The concentration of AEA was significantly elevated in the URB597 post-treated group (P<0.01) compared with the other three groups, including the URB597 pre-treated group. For 2-AG and AA, no difference was identified among all four groups. For PEA, the concentration was significantly higher in the URB597 post-treated group compared with colitis model group ( ${ }^{\#} \mathrm{P}=0.021$ ), while no different was detected between the other groups. These results indicated that only post-treatment with URB597 maintained increased endocannabinoid concentrations. AEA, arachidonoylethanolamide; 2-AG, 2-arachidonoylglycerol; PEA, palmitoylethanolamide; AA, arachidonic acid.

in comparison with the other groups $(\mathrm{P}>0.05)$. Notably, the cognitive functions of the mice were not altered significantly, and the effects of ECS modulation on cognitive function in this murine model remain unknown and require further investigation.

URB597 increases the concentration of AEA but not that of 2-AG in the post-treated group. Following the cognitive function tests, the concentrations of endocannabinoids were determined. As shown in Fig. 5, the concentration of AEA was significantly elevated in the URB597 post-treated group $(\mathrm{P}<0.01)$ compared with the other three groups including the URB597 pre-treated group. For 2-AG and AA, no differences were observed among all four groups. For PEA, the concentration was significantly higher in the URB597 post-treated group compared with the colitis model group $(\mathrm{P}=0.021)$, while no difference was found between the other groups. These results indicated that only post-treatment with URB597 was able to maintain increased concentrations of endocannabinoids. 


\section{Discussion}

In the present study, it was demonstrated by administering URB597, a selective inhibitor of FAAH, that the activation of the ECS was beneficial for the amelioration of TNBS-induced colitis. Compared with the colitis model group, the macroscopic scores of the colitis group were significantly reduced, which is consistent with previous study results (11). An increased 7-day survival rate was also observed in the mice of the URB597 treatment groups, indicating that activation of the ECS was beneficial for the reduction of colitis-induced mortality. In addition, the BBB integrity of the mice was assessed on days 1, 3 and 7 after the induction of colitis. The results suggested that URB597 administration was efficient in maintaining BBB integrity on day 1 after colitis induction. It is noteworthy that no alterations in cognitive functioning were observed in the mice, and the neural protection effects of ECS in this colitis model require further investigation. Notably, only post-treatment (repeated injection) of URB597 was able to maintain increased concentrations of endocannabinoids until day 7; however, pre-treatment (single administration) with URB597 produced some protective effects. This result indicates that early activation of the ECS may be an important cause of these protective effects.

To further examine the protective effect of the ECS against colitis, macroscopic alterations in the colons of the mice were evaluated on days 1, 3 and 7 after colitis induction, and these indicated that the TNBS-ethanol enema steadily induced acute inflammatory colitis. On day 7, the macroscopic changes in the colitis model group were slightly reduced compared with those on days 1 and 3. It is speculated that the reason may be due to the screening mechanism of severe inflammation-induced mortality; specifically, the mice that survived were less susceptible than those that died during the experimental process. However, the results also indicated that URB597 treatment was beneficial for colitis improvement.

Thus, ECS activation not only alleviated TNBS-induced colitis, but also promoted the survival rate of the mice. It was notable that URB597 post-treatment was more efficient than pre-treatment. It is speculated that the post-treatment may chronically stimulate the ECS and sustain a more stable endocannabinoid concentration, which was critical for the relief of colitis symptoms and reduced mortality.

The BBB is a highly selective permeability barrier that allows the passage of nutrients and essential macromolecules to the brain, and prevents the entry of harmful antigens and intestinal bacteria (25). It is well known that severe colitis damages the protective intestinal barrier, subsequently resulting in endoxemia and neural inflammation (6-9). Under inflammatory conditions, it is possible for BBB integrity to be disrupted by the invasion of exogenous toxins (6-9). Therefore, an assessment of BBB integrity was conducted using a dye tracer extraction method in the present study. In this experiment, an increase penetration of Evans blue into the brain tissues was noted, which was suggestive of increased BBB permeability. The results also demonstrated that the administration of URB597 subsequent to colitis induction improved BBB integrity under acute colitis conditions. The potential causes of this improvement are suggested to be as follows: i) The anti-inflammatory effect of endocannabinoids ameliorated the inflammatory response in the inflamed colon and intestinal barrier integrity was maintained, leading to a reduction in the level of harmful substances in the system; ii) the upregulation of endocannabinoids activated central $\mathrm{CB}$ receptors, which was relevant in controlling the BBB integrity.

FCTs were conducted on days 8,9 and 10 to explore the impact of colitis-induced endoxemia on cognitive functions. However, no alteration of cognitive functions was observed, which may be due to the quick recovery of BBB integrity.

In conclusion, the present study investigated the protective role of the ECS in TNBS-induced murine colitis and its associated remote organ lesions, and demonstrated that activation of the ECS effectively reduced the colitis-associated mortality rate and the endoxemia-induced disruption of BBB integrity, indicating that the ECS is a potential target for the treatment of IBD-concomitant impairments.

\section{Acknowledgements}

This study was sponsored by Sichuan Province Science and Technology Support Program (grant no. 2013SZ0030).

\section{References}

1. Di Marzo V, Bifulco M and de Petrocellis: The endocannabinoid system and its therapeutic exploitation. Nat Rev Drug Discov 3: 771-784, 2004.

2. Piomelli D, Giuffrida A, Calignano A and Rodríguezde Fonseca F: The endocannabinoid system as a target for therapeutic drugs. Trends Pharmacol Sci 21: 218-224, 2000.

3. Pertwee RG: Targeting the endocannabinoid system with cannabinoid receptor agonists: Pharmacological strategies and therapeutic possibilities. Philos Trans R Soc Lond B Biol Sci 367: 3353-3363, 2012.

4. Di Marzo V: The endocannabinoid system: Its general strategy of action, tools for its pharmacological manipulation and potential therapeutic exploitation. Pharmacol Res 60: 77-84, 2009.

5. Maccarrone M, Bari M, Battista N and Finazzi-Agrò A: Endocannabinoid degradation, endotoxic shock and inflammation. Curr Drug Targets Inflamm Allergy 1: 53-63, 2002.

6. Di Marzo V and Piscitelli F: Gut feelings about the endocannabinoid system. Neurogastroenterol Motil 23: 391-398, 2011.

7. Alhouayek M and Muccioli GG: The endocannabinoid system in inflammatory bowel diseases: From pathophysiology to therapeutic opportunity. Trends Mol Med 18: 615-625, 2012.

8. Nasser Y, Bashashati M and Andrews CN: Toward modulation of the endocannabinoid system for treatment of gastrointestinal disease: FAAHster but not 'higher'. Neurogastroenterol Motil 26: 447-454, 2014

9. Izzo AA and Sharkey KA: Cannabinoids and the gut: New developments and emerging concepts. Pharmacol Ther 126: 21-38, 2010.

10. Ravikoff Allegretti J, Courtwright A, Lucci M, Korzenik JR and Levine J: Marijuana use patterns among patients with inflammatory bowel disease. Inflamm Bowel Dis 19: 2809-2814, 2013.

11. Engel MA, Kellermann CA, Burnat G, Hahn EG, Rau T and Konturek PC: Mice lacking cannabinoid CB1-, CB2-receptors or both receptors show increased susceptibility to trinitrobenzene sulfonic acid (TNBS)-induced colitis. J Physiol Pharmacol 61: 89-97, 2010.

12. Alhouayek M, Lambert DM, Delzenne NM, Cani PD and Muccioli GG: Increasing endogenous 2-arachidonoylglycerol levels counteracts colitis and related systemic inflammation. FASEB J 25: 2711-2721, 2011.

13. Storr MA, Keenan CM, Emmerdinger D, Zhang H, Yüce B, Sibaev A, Massa F, Buckley NE, Lutz B, Göke B, et al: Targeting endocannabinoid degradation protects against experimental colitis in mice: Involvement of $\mathrm{CB} 1$ and $\mathrm{CB} 2$ receptors. J Mol Med (Berl) 86: 925-936, 2008.

14. Downer EJ: Cannabinoids and innate immunity: Taking a toll on neuroinflammation. ScientificWorldJournal 11: 855-865, 2011. 
15. Fishbein-Kaminietsky M, Gafni M and Sarne Y: Ultralow doses of cannabinoid drugs protect the mouse brain from inflammation-induced cognitive damage. J Neurosci Res 92: 1669-1677, 2014.

16. Sardinha J, Kelly ME, Zhou J and Lehmann C: Experimental cannabinoid 2 receptor-mediated immune modulation in sepsis. Mediators Inflamm 2014: 978678, 2014.

17. Dowie MJ, Howard ML, Nicholson LF, Faull RL, Hannan AJ and Glass M: Behavioural and molecular consequences of chronic cannabinoid treatment in Huntington's disease transgenic mice. Neuroscience 170: 324-336, 2010.

18. Murphy N, Cowley TR, Blau CW, Dempsey CN, Noonan J Gowran A, Tanveer R, Olango WM, Finn DP, Campbell VA and Lynch MA: The fatty acid amide hydrolase inhibitor URB597 exerts anti-inflammatory effects in hippocampus of aged rats and restores an age-related deficit in long-term potentiation. J Neuroinflammation 9: 79, 2012.

19. Henry RJ, Kerr DM, Finn DP and Roche M: FAAH-mediated modulation of TLR3-induced neuroinflammation in the rat hippocampus. J Neuroimmunol 276: 126-134, 2014.

20. Katz PS, Sulzer JK, Impastato RA, Teng SX, Rogers EK and Molina PE: Endocannabinoid degradation inhibition improves neurobehavioral function, blood brain barrier integrity, and neuroinflammation following mild traumatic brain injury. J Neurotrauma 32: 297-306, 2015.
21. Fichna J, Dicay M, Lewellyn K, Janecka A, Zjawiony JK, MacNaughton WK and Storr MA: Salvinorin A has antiinflammatory and antinociceptive effects in experimental models of colitis in mice mediated by KOR and CB1 receptors. Inflamm Bowel Dis 18: 1137-1145, 2012.

22. Wirtz S and Neurath MF: Mouse models of inflammatory bowel disease. Adv Drug Deliv Rev 59: 1073-1083, 2007.

23. Shoji H, Takao K, Hattori S and Miyakawa T: Contextual and cued fear conditioning test using a video analyzing system in mice. J Vis Exp 85: e50871, 2014. doi: 10.3791/50871.

24. Lomazzo E, Bindila L, Remmers F, Lerner R, Schwitter C, Hoheisel U and Lutz B: Therapeutic potential of inhibitors of endocannabinoid degradation for the treatment of stress-related hyperalgesia in an animal model of chronic pain. Neuropsychopharmacology 40: 488-501, 2015.

25. Vendel E and de Lange EC: Functions of the CB1 and CB 2 receptors in neuroprotection at the level of the blood-brain barrier. Neuromolecular Med 16: 620-642, 2014. 